simulation scenarios to explore the relative impact of different intervention approaches across the dementia population.

\section{OP53 LONELINESS, SOCIAL ISOLATION AND ALL-CAUSE MORTALITY IN THE OVER 50S IN IRELAND: FINDINGS FROM THE IRISH LONGITUDINAL STUDY ON AGEING (TILDA)}

${ }^{1} \mathrm{M}$ Ward ${ }^{*},{ }^{1,2} \mathrm{~A}$ Nolan, ${ }^{1}$ RA Kenny. ${ }^{1}$ The Irish Longitudinal Study on Ageing (TILDA), School of Medicine, Trinity College Dublin, Dublin, Ireland; ${ }^{2}$ Economic and Social Research Institute, Dublin, Ireland

\subsection{6/jech-2019-SSMabstracts.54}

Background Interest in loneliness and social isolation as risk factors for premature mortality has recently gained increased attention in both the research literature and public discourses. This has resulted in the established of loneliness taskforces in both Ireland and the United Kingdom. Both loneliness and social isolation have previously been linked to a host of adverse health outcomes, including cardiovascular disease, depression, reduced immune and cognitive functioning, and mortality. Loneliness is most often conceptualised as the subjective assessment of an individual's social relationships while social isolation is the objective quantitative measure of social contacts. Loneliness and social isolation that have been shown to be distinct yet synergistic constructs.

This study examined the association between loneliness, social isolation, and all-cause and cardiovascular mortality in Ireland and also tested the hypothesis that loneliness provides a mechanistic pathway that explains the association between social network size and mortality. This is the first study in Ireland to use linked survey-health assessment-mortality data, combining rich individual-level data from the Irish Longitudinal Study on Ageing (TILDA) with official death certificate data provided by the official mortality register.

Methods The sample included 6,800 participants, including 654 decedents (with 199 deaths due to cardiovascular disease) who had participated in the first round of TILDA. Underlying and contributory causes of death were coded using the International Classification of Diseases, Injuries and Causes of Death (ICD-10). Loneliness was measured using the five-item University of California at Los Angeles (UCLA) Loneliness Scale. Social isolation was measured using the Berkman-Syme Social Network Index. Cox proportional hazards ratios were estimated to assess the association between loneliness, social isolation and mortality while controlling for demographic and socio-economic characteristics, health status, and related behaviours. SEM path analysis was used to identify mechanistic pathways.

Results The average age of death was 78 years. After controlling for other factors, the most lonely (HR 1.39 95\% CI: $1.08-1.78, \mathrm{p}<0.01$ ) and most isolated (HR 1.62 95\% CI: $1.21-2.17, \mathrm{p}<0.001)$ had an increased all-cause mortality risk. A similar association was observed between loneliness and cardiovascular deaths (HR 1.68 95\% CI: 1.06-2.66, $\mathrm{p}<0.05$ ). The association between social isolation and mortality was partially mediated by loneliness.

Conclusion These findings add to the growing body of evidence that loneliness and social isolation are associated with premature mortality. They also shed new light on potential mechanistic pathways that partially explain these associations.

\section{OP54 DESCRIBING CHANGE IN MUSCULOSKELETAL AGING: A COMPARISON OF TECHNIQUES USING DATA FROM THE HEALTH, AGING AND BODY COMPOSITION STUDY}

LD Westbury*, HE Syddall, EM Dennison, C Cooper. MRC Lifecourse Epidemiology Unit, University of Southampton, Southampton, UK

\subsection{6/jech-2019-SSMabstracts.55}

Background Preventive strategies for musculoskeletal disorders require a better understanding of age-related changes in muscle strength, physical function and body composition (including bone). Many publications use simple change measures from observations at two time-points which do not account for measurement error and assumes change is linear. Sophisticated techniques for analysing change are available but are rarely implemented in this field.

Methods Changes in grip strength, walking speed, lean mass and hip bone mineral density (BMD) were explored among 3075 men and women from the Health, Aging and Body Composition Study; each measure was assessed at least 5 times during a median 9 year follow-up period. The following techniques were implemented: linear mixed effects models (LMEM) (applied to raw data and age-specific z-scores from generalised additive models for location, scale and shape); growth mixture models (GMM); and latent class trajectory models (LCTM). LMEM use random effects to capture interindividual variation in level and change around a populationaverage trajectory; GMM extend LMEM by identifying clusters of individuals with similar trajectories and deriving cluster-specific average trajectories; and LCTM are simplified GMM with no random effects, assuming all individuals in a cluster have the same trajectory.

Results Mean (SD) age at baseline was 74.1 (2.9) years. Mean annual percentage declines for walking speed and grip strength were $2.1 \%$ and $1.5 \%$ respectively; declines were smaller for hip BMD (0.6\%) and lean mass (0.5\%). Trajectories from LMEM (applied to raw data) for grip strength, walking speed and hip BMD were quadratic in relation to age such that declines accelerated with advancing age; decline in lean mass was linear. Random slopes from LMEM applied to Z-scores were weakly correlated with baseline levels for all characteristics $(-0.36<\mathrm{r}<0.17)$, resulting in person-specific measures of change that were broadly independent of level. All GMM contained a group comprising at least $80 \%$ of the sex-specific sample with sparse numbers of participants in other groups, suggesting that a LMEM with a single population average trajectory describes most of the change in the sample. LCTM derived subgroups with much larger differences in levels of the characteristics rather than in rates of loss.

Conclusion LMEM enable a more comprehensive analysis of change compared to methods using data from only two timepoints. However, inter-individual differences in rates of change regarding musculoskeletal parameters in this age group and duration of follow-up may be too small to be identified using more complex techniques such as GMM or LCTM. 